\title{
Du Dicoumarol aux Anti-Vitamines K Artificielles
}

\author{
Par P. Meunier
}

Laboratoire de Chimie Biologique de la Faculté des Sciences, 93 Rue Pasteur, Lyon

C'est en r 941 que Link et ses collaborateurs aux Etats-Unis nous ont fait connaitre la constitution chimique de l'agent responsable de la curieuse maladie dite du 'mélilot gâté'. Il s'agit d'accidents hémorragiques qui surviennent chez le bétail, les lapins, etc. qui ingèrent ce fourrage. Voici la formule développée du composé toxique appelé dicoumarol par les auteurs américains:<smiles>O=c1oc2ccccc2c(O)c1Cc1c(O)c2ccccc2oc1=O</smiles>

Méthylène-3: $3^{\prime}$-bishydroxy-4:4'-coumarine (Stahmann, Huebner \& Link, 1941)

On ne peut manquer de faire un rapprochement entre la structure chimique de ce composé et celle de la méthyl-2-naphto-hydroquinone par exemple qui est une vitamine $\mathrm{K}$ :

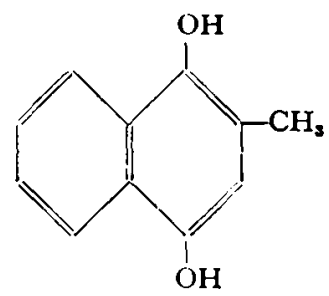

On était déjà à l'époque sous l'influence des idées qui venaient de se faire jour en physiologie microbienne selon lesquelles une modification parfois légère apportée à la molécule d'une vitamine ou d'un facteur de croissance pouvait aboutir à un produit d'activité inverse, un antagoniste de la croissance microbienne (Fildes, 1940; Kuhn, 1942).

Ce fut pour nous l'occasion (avec C. Mentzer) de rechercher si ce qu'on a appelé fort justement des anti-vitamines était une réalité valable aussi pour les animaux supérieurs. Le dicoumarol devait-il être considéré comme une antivitamine $\mathrm{K}$ ? La spécificité physiologique très étroite de ce toxique semblait déjà plaider en faveur d'une réponse positive; en effet, son action consiste uniquement en la baisse de la prothrombine du sang, c'est à dire qu'elle est exactement opposće à celle d'une vitamine $\mathrm{K}$, sans qu'aucune autre fonction physiologique soit atteinte.

Quant à l'antagonisme entre la vitamine et l'anti-vitamine en qui devait résider la clé du problème, nous l'avions constaté dès 1942 (Meunier \& Mentzer, 1942); mais il a été longtemps discuté. Il est assez instructif de rechercher aujourd'hui les raisons des incertitudes antérieures. L'une de ces raisons était établie sur le fait qu'il suffit de très peu de vitamine $\mathrm{K}$, des quantités que le lapin par exemple trouve normalement dans ses putréfactions intestinales, pour rétablir, chez le mammifère intoxiqué, un taux de 
prothrombine normale au fur et à mesure que le dicoumarol s'élimine de l'organisme (Fig. I). Par contre, de très grosses quantités de vitamine $\mathrm{K}$ sont nécessaires pour améliorer dès le début de l'intoxication au dicoumarol le taux de prothrombine de l'animal (Fig. 2).

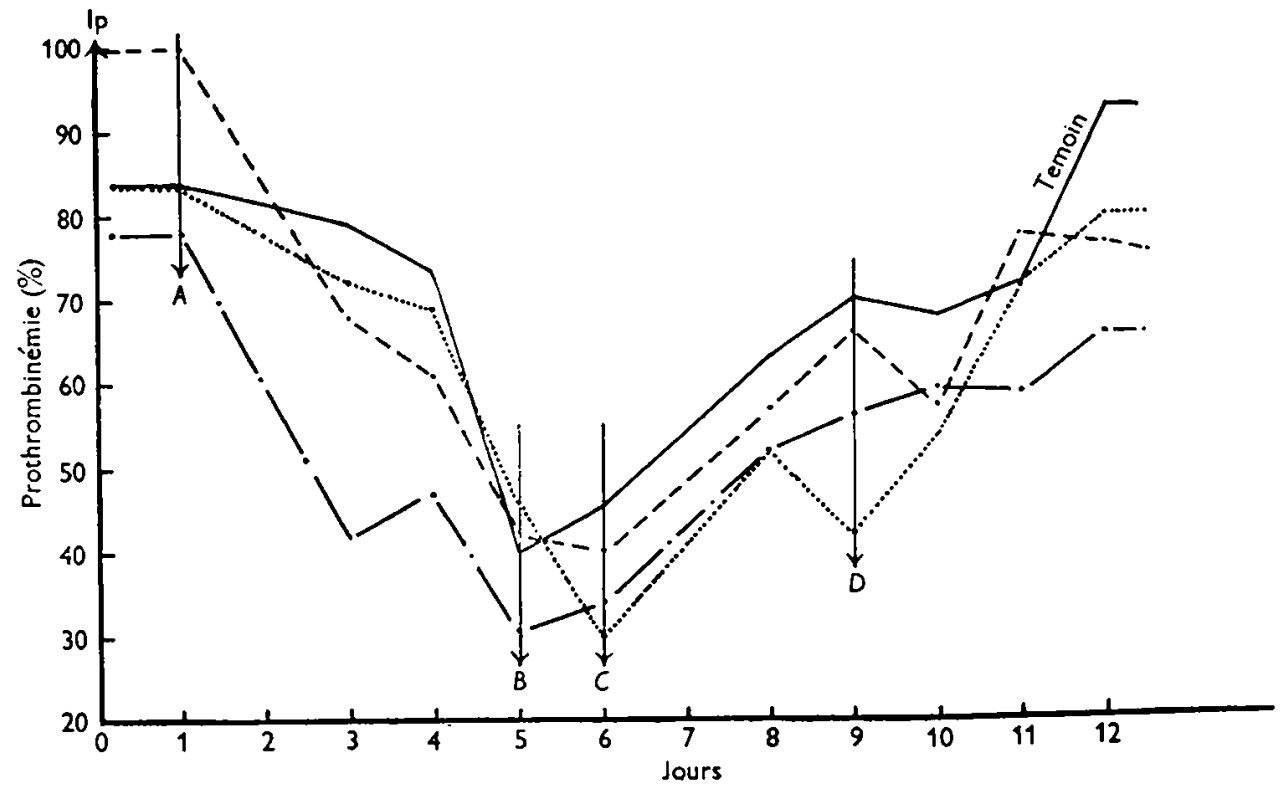

Fig. 1. Effet du dicoumarol et de la méthyl-2-naphtoquinone sur la prothrombinémie des lapins. $A, 1 \mathrm{mg} .{ }_{i k g}$. de dicoumarol aux quatre lapins; $B, 2.5 \mathrm{mg} . / \mathrm{kg} . ; C, 3.5 \mathrm{mg} .{ }^{\mathrm{kg}}$; $D, 7 \mathrm{mg} . / \mathrm{kg}$. de méthyle-2-naphtoquinone aux trois lapins (d'après Fournel \& Bovet, I943).

L'antagonisme entre le dicoumarol et la vitamine $\mathrm{K}$ est aujourd'hui admis par tous et l'on sait qu'il ne peut se manifester qu'après des doses relativement faibles de dicoumarol, voir par exemple les excellentes revues de Quick (1944) et de Woolley (r947), et sous l'effet d'au moins sept parties en poids de vitamine K.

Lorsque nous avons réussi à préparer, dans la série de la naphtoquinone elle-même, ce que l'on pourrait appeler des anti-vitamines $\mathrm{K}$ artificielles, la démonstration de l'antagonisme avec la vitamine $\mathrm{K}$ devint très facile et indiscutable (Meunier, Mentzer, Buu Hoï \& Cagniant, 1943); ce fut le cas notamment avec la méthylène-bis-hydroxynaphtoquinone que nous avons nommée le di-phtiocol.<smiles>CC1=C(O)C(=O)c2ccccc2C1=O</smiles>

Phtiocol, vitamine $\mathrm{K}$

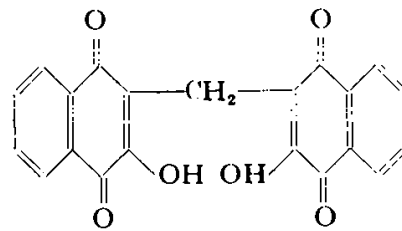

di-phtiocol, anti-vitamine $\mathrm{K}$

Ce nouveau venu, il est vrai, est beaucoup moins actif que le dicoumarol lui-même, mais il est très intéressant de rapprocher son action chez l'animal de celle du poison 
hemorragique du melilot gâte. On sait qu'on peut obtenir avec ce dernier des prothrombinemies abaissees a moins de $1 \%$ de la normale, chez le lapin; mais il faut 4 jours pour obtenir ce résultat, après $24 \mathrm{hr}$. la prothrombinémie etant encore a $20 \%$ environ (cf. Quick, 1944). De plus, au-dessus d'une certaine dose critique de dicoumarol, les augmentations même considérables de posologie n'accélèrent pas la chute de

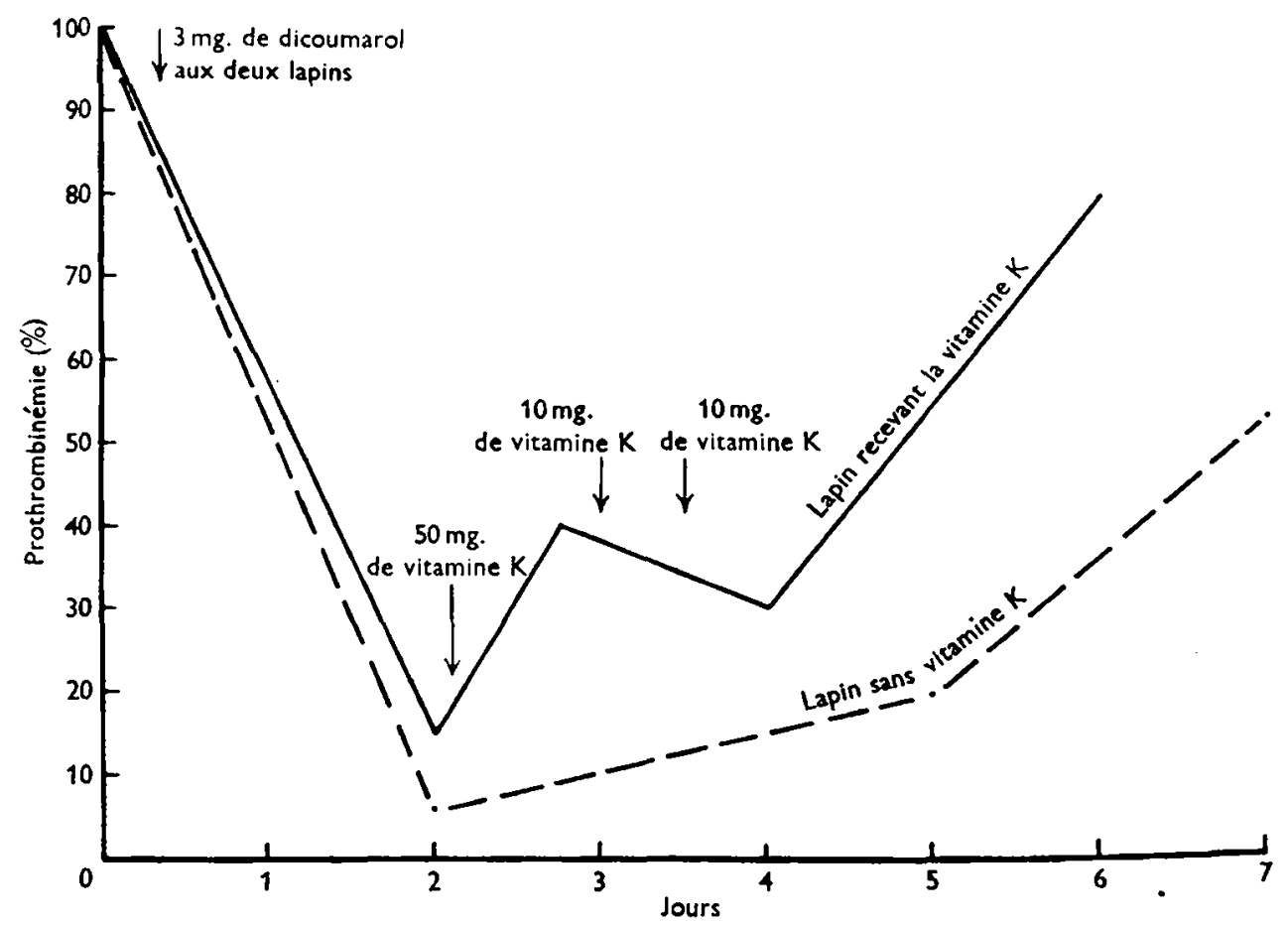

Fig. 2. Effet de la vitamine $\mathrm{K}$ sur la prothrombinémie du lapin au cours de l'intoxication au dicoumarol.

la prothrombine (Quick, 1944). L'existence d'une dose limite, nous l'avons bien mise en évidence aussi dans le cas du di-phtiocol. Nous l'avons retrouvée également avec une autre anti-vitamine $\mathrm{K}$ appartenant à une série chimique tout à fait différente (Meunier, Mentzer \& Molho, 1947), la phényle-indanedione,<smiles>O=C1c2ccccc2C(=O)C1c1ccccc1</smiles>

Bien qu'il y ait parfois de grandes différences d'un animal à l'autre, il semble que ni le di-phtiocol, ni la phényle-indanedione ne puissent abaisser au-dessous de $20 \%$ le taux de prothrombine, quelle que soit la dose utilisée, et cet effet est obtenu en $24-48 \mathrm{hr}$. Et si l'on compare de façon plus précise les courbes d'action sur la prothrombine du lapin par exemple des différents produits précédemment cités, on constate que la vitesse de chute de la prothrombine varie peu avec la nature du produit utilisé; mais 
cette chute s'arrête beaucoup plus vite dans le cas de nos anti-vitamines $\mathrm{K}$ artificielles moins actives (Fig. 3). Ceci nous amène à dire que l'élimination du produit toxique commande tout le phénomène. Tout se passe comme si l'anti-vitamine $\mathrm{K}$ se bornait à empêcher pendant plus ou moins longtemps, selon les conditions de son élimination

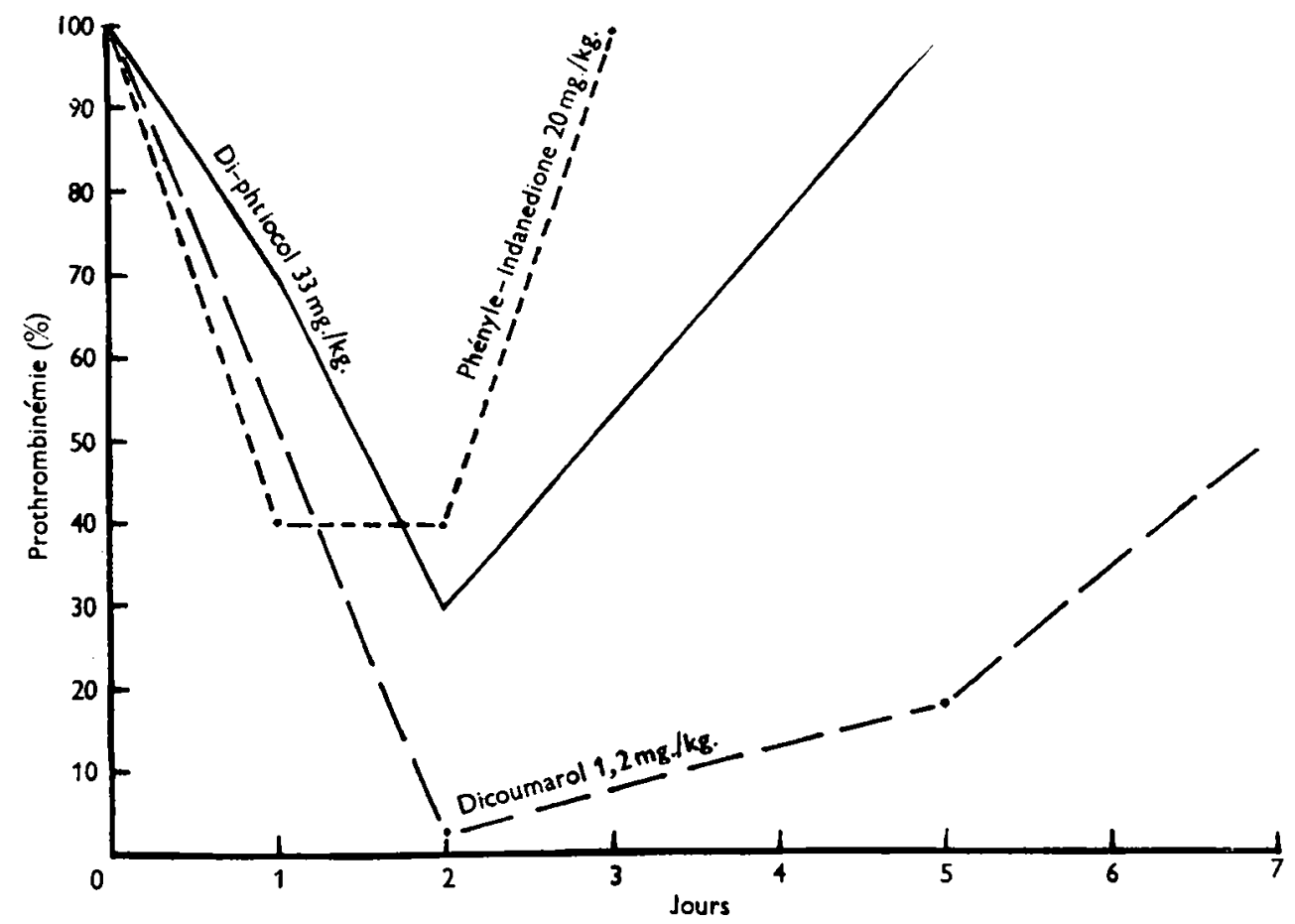

Fig. 3. Effet des anti-vitamines $\mathrm{K}$ artificielles sur la prothrombinémie du lapin.

par l'organisme, la formation de la prothrombine. Comme l'a dit très justement Quick (1944) à propos du dicoumarol 'la chute de la prothrombine observée représente réellement la consommation de prothrombine ou sa destruction par l'organisme animal'.

Il est vrai que les cliniciens ne se sont pas contentés de la simple hypo-prothrombinémie pour rendre compte de l'emploi favorable du dicoumarol contre les thromboses. Selon les observations de Bingham, Meyer \& Pohle (I941), le diamètre des capillaires se dilate par des doses massives de dicoumarol. Dale \& Jaques (1942) ont attiré l'attention sur la perturbation apportée par le dicoumarol à l'agglutination des plaquettes sanguines. Enfin, on a observé une action de certaines anti-vitamines $\mathrm{K}$ sur la fragilité capillaire. La phényle-indanedione abaisse notablement cette fragilité selon Gley \& Delor (1948).

Pour s'en tenir au point de vue purement biochimique, on peut dire que les antivitamines $\mathrm{K}$ sont des substances qui interrompent la synthèse par l'organisme de la prothrombine, et cela pendant plus ou moins longtemps. L'hypo-prothrombinémie qui en résulte est alors plus ou moins accentuée. Si des hypothèses comme celle de Lyons (1945) se vérifiaient, le problème pourrait être un peu déplacé: on pourrait 
penser à un effet direct de l'anti-vitamine sur les groupements $\mathrm{SH}$ du fibrinogène. Dans tous les cas, c'est du progrès de nos connaissances sur des protéines spécifiques, la prothrombine ou le fibrinogène, que viendra l'explication du mécanisme intime de l'action des anti-vitamines $\mathrm{K}$ chez l'animal.

Mais aujourd'hui la notion d'anti-vitamine $\mathrm{K}$ déborde singulièrement le cadre de la physiologie animale. De nombreux anti-biotiques, fungicides etc. ont été préparés, qui sont plus ou moins apparentés par leur structure à la méthyle-2-naphtoquinone et dont l'action est inhibée par cette dernière. Citons notamment l'iodinine (McIlwain, r943), la dichloro-2:3-naphtoquinone (Woolley, 1945) et les dérivés monochlorés de la naphtoquinone et de l'hydroxy-2-naphtoquinone étudiés dans notre laboratoire (Guérillot-Vinet, I948).

Il faut remarquęr d'ailleurs qu'il n'y a pas de parallélisme entre l'activité hypoprothrombinémiante et l'activité anti-bactérienne de ces corps. Ainsi le dicoumarol lui-même, le plus actif de tous chez l'animal, est relativement peu actif comme antibactérien (Maghski, Copley \& Couch, r947).

Il est vrai que la dernière venue dans la liste des anti-vitamines $\mathrm{K}$, une substance d'origine naturelle, la méthoxy-2-naphtoquinone (Little, Sproston \& Foote, 1948), est à la fois un anti-biotique puissant vis-à-vis par exemple d'un grand nombre de champignons parasitaires des végétaux et un abaisseur de prothrombine chez l'animal (Molho, Moraux \& Meunier, 1948).

Ce dernier exemple nous ramène à des préoccupations de nutritionistes. Il nous indique que la question des anti-vitamines $\mathrm{K}$ dans la nutrition animale n'est certainement pas close avec la connaissance du dicoumarol.

\section{BIBLIOGRAPHIE}

Bingham, J. B., Meyer, O. O. \& Pohle, F. J. (1941). Amer. F. med. Sci. 202, 563 .

Dale, D. L. \& Jaques, L. B. (1942). Canad. med. Ass. F. 46, 546.

Fildes, P. (1940). Lancet, 238, 955.

Fournel, J. \& Bovet, D. (1943). Bull. Soc. Chim. biol., Paris, 25, 390.

Gley, P. \& Delor, J. (1948). Bull. Soc. Chim. biol., Paris. (A paraitre.)

Guérillot-Vinet, A. (1948). Bull. Soc. Chim. biol., Paris. (À paraître.)

Kuhn, R. (1942). Chemie, p. I.

I ittle, J. E., Sproston, T. J. \& Foote, M. W. (1948). F. biol. Chem. 174, 335.

Lyons, R. N. (1945). Nature, Lond., 155, 633.

McIlwain, H. (1943). Biochem. F. 37, 265.

Maghski, J., Copley, M. J. \& Couch, J. F. (1947). Science, 105, 125.

Meunier, P. \& Mentzer, C. (1942). Bull. Soc. Chim. biol., Paris, 24, 371.

Mcunier, P., Mentzer, C., Buu Hoï \& Cagniant (1943). Bull. Soc. Chim. biol., Paris, 25, 384.

Meunier, P., Mentzer, C. \& Molho, D. (1947). C.R. Acad. Sci., Paris, 224, 1666.

Molho, D., Moraux, J. \& Meunier, P. (1948). Bull. Soc. Chim. biol., Paris, 30, 637.

Quick, A. J. (1944). Physiol. Rev. 24, 297.

Stahmann, M. A., Huebner, C. F. \& Link, K. P. (1941). Y. biol. Chem. 138, 513 .

Woolley, D. W. (1945). Proc. Soc. exp. Biol., N.Y., 60, 225.

Woolley, D. W. (1 947). Physiol. Rev. 27, 308. 